\title{
Sodium metabisulfite hypersensitivity in urticaria
}

\section{Beata Sadowska, Marlena Sztormowska, Marika Gawinowska, Marta Chelminska}

\author{
Department of Allergology, Allergology and Pneumonology Clinic, Medical University of Gdansk, Smoluchowskiego 17, 80- \\ 214 Gdansk, Poland
}

Corresponding author: Beata Sadowska, MD, E-mail: sadowska.beata@yahoo.pl

\begin{abstract}
Background: Sodium metabisulfite is a recognized, but rare, trigger of urticaria, wherein the IgE mechanism has been sporadically proven. The aim of this study was to identify the potential reaction to sodium metabisulfite (MBS) based on a placebo-controlled oral challenge in patients with urticaria and suspected hypersensitivity to food additives. Materials and Methods: A total of 110 adult patients (76 females and 34 males with a mean age of 46 years) were included in the study between 2017 and 2019. All subjects underwent MBS skin prick tests (SPT) and patch tests (PT). Patients with a positive skin test or suspected MBS hypersensitivity were qualified for a placebo-controlled oral challenge (OC). Results: Skin testing was positive in 24 patients: SPT in 20\% ( $n=22)$, PT in 5\% $(n=6)$. Out of 64 oral challenges, 13 positive results were obtained. Patients with a positive challenge typed sulfite foods twice as often as a culprit compared to those with a negative OC. Conclusions: In patients with urticaria, both the IgE and non- $\operatorname{IgE}$ mechanism of MBS hypersensitivity has been demonstrated. Skin tests with a detailed medical history of potentially guilty foods may be helpful in determining sulfite hypersensitivity.
\end{abstract}

Key words: Sulfites; Sodium metabisulfite; Urticaria; Food additive hypersensitivity

\section{INTRODUCTION}

Sulfites are a group of inorganic salts that, in a certain environment, liberate sulfur dioxide. A range of themsuch as sodium and potassium metabisulfite, sodium sulfite, and sodium and potassium bisulfite-are used in the food, beverage, cosmetic, medicine, rubber, and photographic industry as a preservative and a bleaching and antioxidative agent [1-3]. They are key preservatives of wine, beer, grapefruit juice, dried fruits, and seafood and are widely used in the production of processed foods [4-6]. The acceptable daily intake (ADI) for sulfites, in terms of sulfur dioxide, is $0.7 \mathrm{mg}$ per $1 \mathrm{~kg}$ of body weight per day, but their intake is often exceeded. According to the European Union regulation, the total amount of sulfur dioxide in wine must not exceed $150 \mathrm{mg} / \mathrm{L}$ for red wines and $200 \mathrm{mg} / \mathrm{L}$ for white and rose wines, and is higher for sparkling and sweet wines (up to 235 and $400 \mathrm{mg} / \mathrm{L}$ respectively). Although producers are obliged to label products with a sulfite concentration of over $10 \mathrm{mg} / \mathrm{L}$ appropriately, they are not obliged to disclose the total amount of the substance [5].

The hypersensitivity symptoms described after sulfites ingestion may include rhinitis [7], asthma [6,8-10], urticaria [11-13], systemic rash [14-16], intestinal disorders, dizziness [7], headache [17], and anaphylaxis [7,18-20]. Sulfites are thought to be the main food additive exacerbating asthma, which affects $3-10 \%$ of the population [20]. Numerous studies have reported sensitization to take place through the skin, for instance, after the application of an anti-fungal or anti-hemorrhoid medicine, hair dye, or facial cream or after contact with animal feed, caused by prolonged occupational exposure $[7,20]$. Contact allergy is also a proven and well-known sulfite side effect $[1,7,21-23]$.

The mechanisms lying behind these reactions are only partially known [24]. Some of the patients may not be able to metabolize sulfiting agents well enough due to a deficiency of the enzyme sulfite oxidase, which

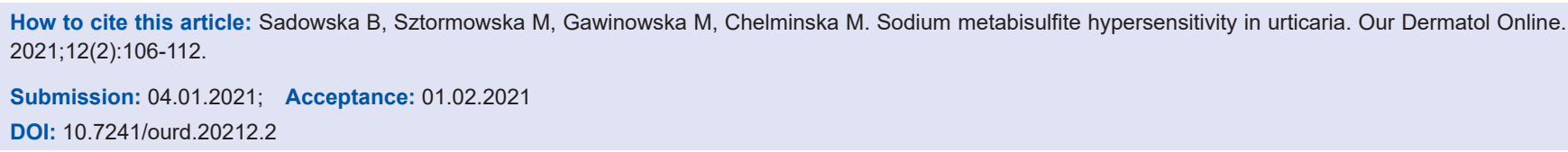


may lead to hypersensitivity symptoms $[9,20]$. An interesting thesis is the possibility of suppression of the Thl-type immune response by sulfites, resulting in the advantage of the Th2 mechanism [25]. The immunological background of the reaction has been proven in some cases [26], but other authors question this link [4].

Several observations indicate that the asthma phenotype is more common than urticaria in sulfite hypersensitivity $[5,8,9]$. The subject of the present study was to assess the frequency of sodium metabisulfite hypersensitivity in patients with urticaria and suspected hypersensitivity to food additives, with an identification of the $\operatorname{IgE}$ and non-IgE dependent reactions.

\section{MATERIALS AND METHODS}

\section{Patients}

The study included patients with a duration of urticaria of more than six weeks, hospitalized with a suspicion of hypersensitivity to food additives, at the Department of Allergology of the Medical University of Gdańsk, Poland, between 2017 and 2019. A group of 110 patients was qualified for the study (Table 1). Before hospitalization, all participants completed a detailed questionnaire regarding the duration and nature of urticaria, eating habits, the use of an elimination diet, the allergy diagnostics performed, as well as the assessment of the effectiveness of the current treatment.

Patients had not been taking antihistamines for seven days and systemic glucocorticoids for one month prior to hospitalization. Spirometry was performed for any coexisting asthma or chronic obstructive pulmonary disease. Written consent was obtained. The study protocol was approved by the local bioethics committee (NKBBN/546/2016-2017) and was in line with the principles of ethics and the Declaration of Helsinki.

\section{Skin Testing}

All patients underwent MBS skin tests. Sodium metabisulfite from Euro-Win Ltd., Jaslo, Poland, was used in the study. To prepare SPTs, it was diluted with saline and glycerol at a 1:1 ratio to obtain a $1 \%$ solution (i.e., a concentration of $10 \mathrm{mg} / \mathrm{mL}$ ). A positive and negative control was performed (histamine hydrochloride $10 \mathrm{mg} / \mathrm{mL}$ and saline from Allergopharma). A wheal diameter of a minimum
Table 1: Patient characteristics.

\begin{tabular}{|c|c|c|}
\hline Number of patients & & $n=110$ \\
\hline Average age (in yrs.) & & 46.1 \\
\hline Females / males & & $69 \% / 31 \%$ \\
\hline Atopy & & $64.6 \%$ \\
\hline Asthma & & $22.7 \%$ \\
\hline Systemic symptoms & & $56.4 \%$ \\
\hline Past use of glucocorticoids & & $42.7 \%$ \\
\hline Adrenaline prescription & & $40.9 \%$ \\
\hline Manifestation of urticaria & Urticaria + angioedema & $50.9 \%$ \\
\hline & Urticaria only & $32.7 \%$ \\
\hline & Angioedema only & $16.4 \%$ \\
\hline Duration of disease & Average duration (in yrs.) & 7.2 \\
\hline & Up to 5 years & $53.6 \%$ \\
\hline & More than 5 years & $46.4 \%$ \\
\hline Onset of symptoms* & More often at night & $26.4 \%$ \\
\hline & Until noon & $4.6 \%$ \\
\hline & In the afternoon & $19.1 \%$ \\
\hline & Other & $58.2 \%$ \\
\hline Symptoms location* & Face & $83.6 \%$ \\
\hline & Hands & $36.4 \%$ \\
\hline & Forearms and arms & $52.7 \%$ \\
\hline & Trunk & $52.7 \%$ \\
\hline & Feet & $34.6 \%$ \\
\hline & Legs & $39.1 \%$ \\
\hline & Genitals & $20.9 \%$ \\
\hline $\mathrm{AH} 1$ treatment efficacy & Good & $22.7 \%$ \\
\hline & Partial & $35.5 \%$ \\
\hline & Bad & $41.8 \%$ \\
\hline
\end{tabular}

* Multiple responses were allowed.

of $3 \mathrm{~mm}$ with surrounding erythema larger than the negative control and representing the average of the two largest perpendicular dimensions was considered positive. A negative MBS SPT was obtained in 100 patients from the control group without urticaria who were consulted at the outpatient clinic.

Patch tests were performed with commercial sodium metabisulfite (S-011, Chemotechnique Diagnostics, Vellinge, Sweden), and MBS on a petrolatum vehicle prepared by the pharmacy, both in a $1 \%$ concentration. The tests were performed using IQ-Ultra chambers (Chemotechnique Diagnostics, Vellinge, Sweden) and rated on days D2, D3, D4, D5, D6, and D7. Readings were performed according to the International Contact Dermatitis Research Group criteria. Some of the readings were carried out on an outpatient basis. An intravenous line single-blind oral challenge with MBS was then performed.

\section{Oral Challenge}

Patients with any positive skin test or a history suggesting the possibility of sulfite hypersensitivity were qualified for a single-blind placebo-controlled 
oral challenge. OCs were performed according to the following protocol: placebo, $10 \mathrm{mg}, 20 \mathrm{mg}$, and $100 \mathrm{mg}$ capsules were administered in 30-minute intervals, followed by $200 \mathrm{mg}$ after two hours. After the last dose, the patients underwent a minimum of two hours of observation, and immediate contact with the hospital was recommended within the next 24 hours should any symptoms appear. A test was considered positive according to objective symptoms, such as urticaria, angioedema, conjunctivitis, rhinitis, and bronchoconstriction confirmed in spirometry within 24 hours after the challenge. In the case of a hypersensitivity reaction, the participants were given antihistamines, glucocorticoids, and intramuscular adrenaline as needed.

\section{Statistical Analysis}

The significance of the association among variables was analyzed with a chi-squared test and Fischer's exact test. $P$ values below .05 were considered significant.

\section{RESULTS}

\section{Questionnaire}

Almost every second patient (48\%) experienced symptoms less than once a month, while $16 \%$ reported the frequency of urticaria from eight to thirty days. Based on this data and on medical history, we classified $65 \%$ of the cases as chronic inducible urticaria (CindU), $57 \%$ as chronic spontaneous urticaria (CSU), and 22\% into the CindU + CSU group. Atopy, defined as a minimum of one positive skin prick test or serum IgE for airborne or food allergens, was established in $65 \%$ of the subjects. Seventy percent of the patients were convinced that it was the food that caused the symptoms. The following foods, potentially with sulfites, were listed as guilty by 24 patients before the study: wine, beer, dried fruits, olives, cakes, herring fillets, and restaurant meals.

\section{Skin Prick Test (SPT)}

A positive sodium metabisulfite SPT was obtained in $20 \%$ of the participants (MBS SPT $(+), \mathrm{n}=22$ ), among which one result was prolonged with a maximum skin reaction observed only after 3.5 hours (Fig. 1). Unlike the MBS SPT (-) patients, no statistically significant difference in sex, asthma, and atopy prevalence was observed in the MBS SPT $(+)$ patients. The location of urticarial symptoms was more common in the lower limbs in the MBS SPT $(+)$ patients than in the MBS SPT (-) (59 vs. 34\%, $p<.032)$. Out of the 20 patients with a positive skin prick test, the oral challenge was positive in $40 \%(\mathrm{n}=8, p<.009)$.

\section{Patch Test (PT)}

Although patch tests are not a feasible test for the diagnosis of urticaria per se, they may show evidence of concomitant type I and type IV allergy reactions. A positive patch test (MBS PT $(+))$ was noted in six patients (5.5\%; 5 females and 1 male). The tests were positive on D3 through $\mathrm{D} 5$ in all six patients and up to D7 in two. The results were similar on both vehicles, commercial and pharmacy prepared (Fig. 2). All six patients had a history of angioedema and urticaria, and three of them suffered from throat edema in the

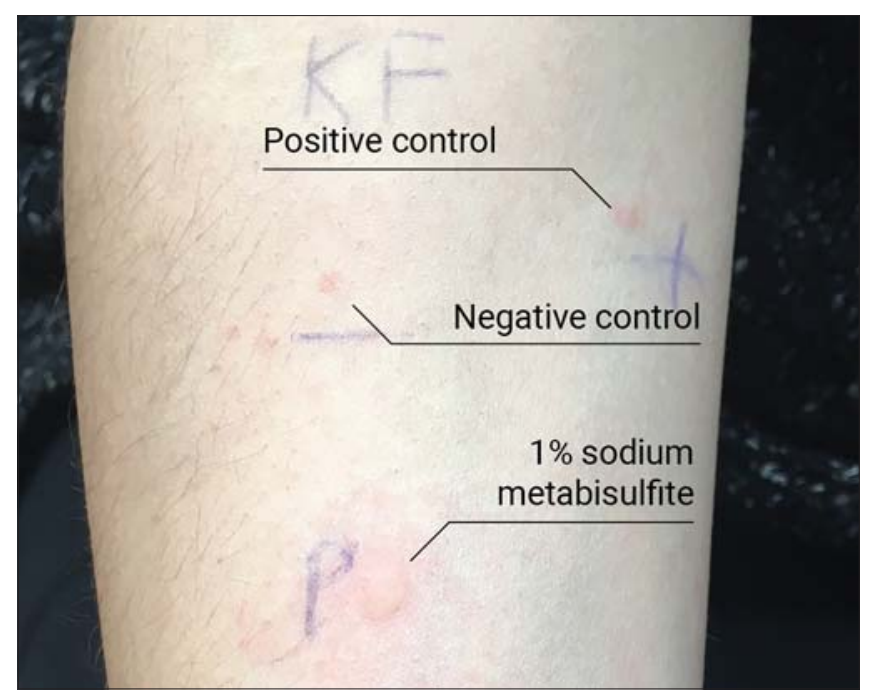

Figure 1: Sodium metabisulfite (MBS) skin prick test (SPT) after 3.5 hours.

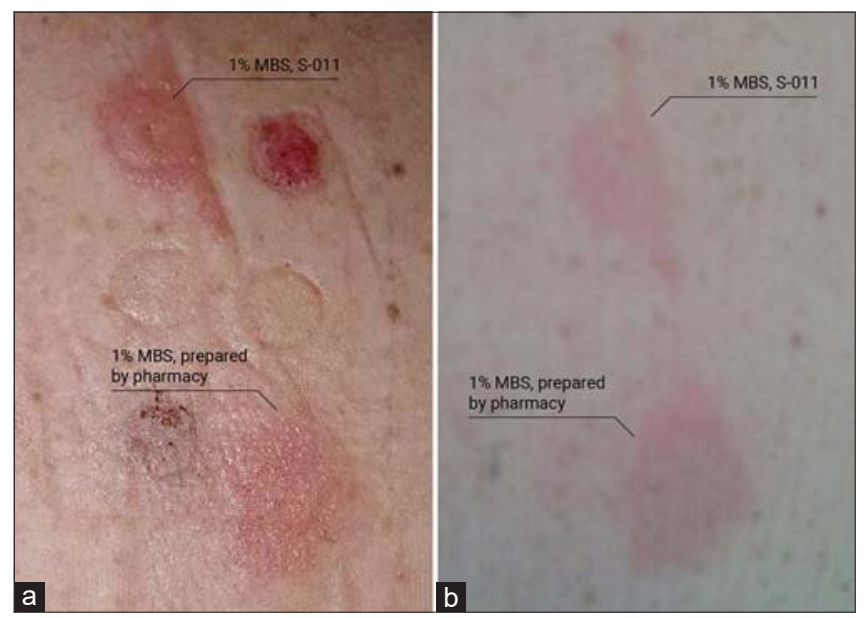

Figure 2: Positive sodium metabisulfite patch tests (PT) (a) after 48 hours and (b) after 120 hours. 
past. In this group, next to the typical facial location, compared to the group with a negative patch test, urticaria symptoms more often affected the hands ( $83 \%$ vs. $34 \% ; p<.015)$, feet ( $83 \%$ vs. $32 \% ; p<.012$ ), and genitals $(67 \%$ vs. $18 \% ; p<.013)$. This group was dominated by people over 50 years of age and their complaints had persisted for over five years $(n=5$; $83 \%$ ). In addition, as many as five patients had used parenteral glucocorticoids ( $83 \%$ vs. $40 \%$; $p<.039$ ). In this group, the sodium metabisulfite SPT was positive in four subjects $(67 \% ; p<.033)$, and the challenge test was positive in 2 out of the 6 patients.

\section{Oral Challenge (OC)}

An oral challenge with sodium metabisulfite was carried out in 64 out of the 110 patients. Two MBS SPT (+) patients were not challenged; one refused to participate while the other had contraindications (forced expiratory volume in one second (FEV1) below $70 \%)$. A positive result was obtained in 13 patients (MBS OC $(+)$ ).

Reactions occurred with $10 \mathrm{mg}, 20 \mathrm{mg}, 100 \mathrm{mg}$, and $200 \mathrm{mg}$ doses in one, three, four, and five patients, respectively. These included urticaria, angioedema, rhinoconjunctivitis, shortness of breath not supported by spirometry, flatulence, pruritus, tachycardia, headache, and weakness (Table 2). All these patients were treated with a double antihistamine dose (prednisone in three and intramuscular adrenaline in two). All objective symptoms resolved within two hours of intervention.

Unlike the MBS OC (-), the MBS OC (+) patients slightly more often indicated that the food was the culprit ( $92 \%$ vs. $74 \%$; $p<.17$ ), including $46 \%$ with complaints about food containing potential sulfites. A subsequent analysis of the causative agents reported in the questionnaires showed that the MBS OC (+) patients, unlike the MBS OC (-), more often indicated that physical exertion induced urticaria (38\% vs. $12 \%$; $p<.023)$. Alcohol, stress, heat, dermographism, infection, prolonged pressure, water, sweating, vibrations, sex cycle, and drugs were similarly often a reaction cofactor in both groups, while cold and UV radiation were slightly more often indicated as the cofactor (23\% vs. $10 \%$ in both groups; $p$ insignificant). The MBS SPT was positive in $62 \%$ of the patients with a positive challenge, and only in $24 \%$ of those with a negative challenge $(p<.009)$.

\section{DISCUSSION}

In our study, we determined that hypersensitivity to sodium metabisulfite occurred in $12 \%$ of the studied patients with urticaria and suspected to have hypersensitivity to food additives. We confirmed that the clinical significance of sulfites is underestimated [20].

Several mechanisms are thought to play a role in the observed sulfites hypersensitivity, but $\operatorname{IgE}$ mediated reactions have rarely been documented so far $[11,27,28]$.

In the current study, we demonstrated that, in patients with urticaria and MBS hypersensitivity, the underlying IgE mechanism is as common as the non-IgE. Assuming that a positive SPT result in an MBS OC $(+)$ patient is evidence of IgE-dependent reactions, we confirmed the allergic mechanism in $62 \%$ of the patients ( 8 out of the 13) with a hypersensitivity reaction to MBS.

In our study, we noted a relatively high percentage of MBS positive skin prick tests $(20 \%)$ in patients with urticaria. This finding may be explained by the strict selection of patients with suspected hypersensitivity to food additives, wherein most of them suffered from CindU.

Yang et al. obtained positive skin tests with potassium metabisulfite in five patients, which correlated with three positive challenges out of four carried out among 35 patients with hypersensitivity reactions after restaurant meals, wine, or drinks [18].

Reports of hypersensitivity to sulfites in urticaria are divergent. Jimenes-Aranda et al. found hypersensitivity to sodium metabisulfite, sodium bisulfite, and potassium bisulfite in $36 \%, 33 \%$, and $31 \%$ of patients, respectively [28]. Montaño García and Orea reported a positive response after an MBS challenge in $6 \%$ of patients [29]. On the other hand, Rajan et al. showed that after the administration of various food additives, including $100 \mathrm{mg}$ of MBS, patients with urticaria experienced no symptoms in a double-blind challenge [30]. One's country's eating habits and the actual sulfite content in the foods available may be important. A patient from the UK was described complaining of a rash as a result of diet changes after a visit to Italy, such as the consumption of seafood, wine, grapes, and fries [15]. 
www.odermatol.com

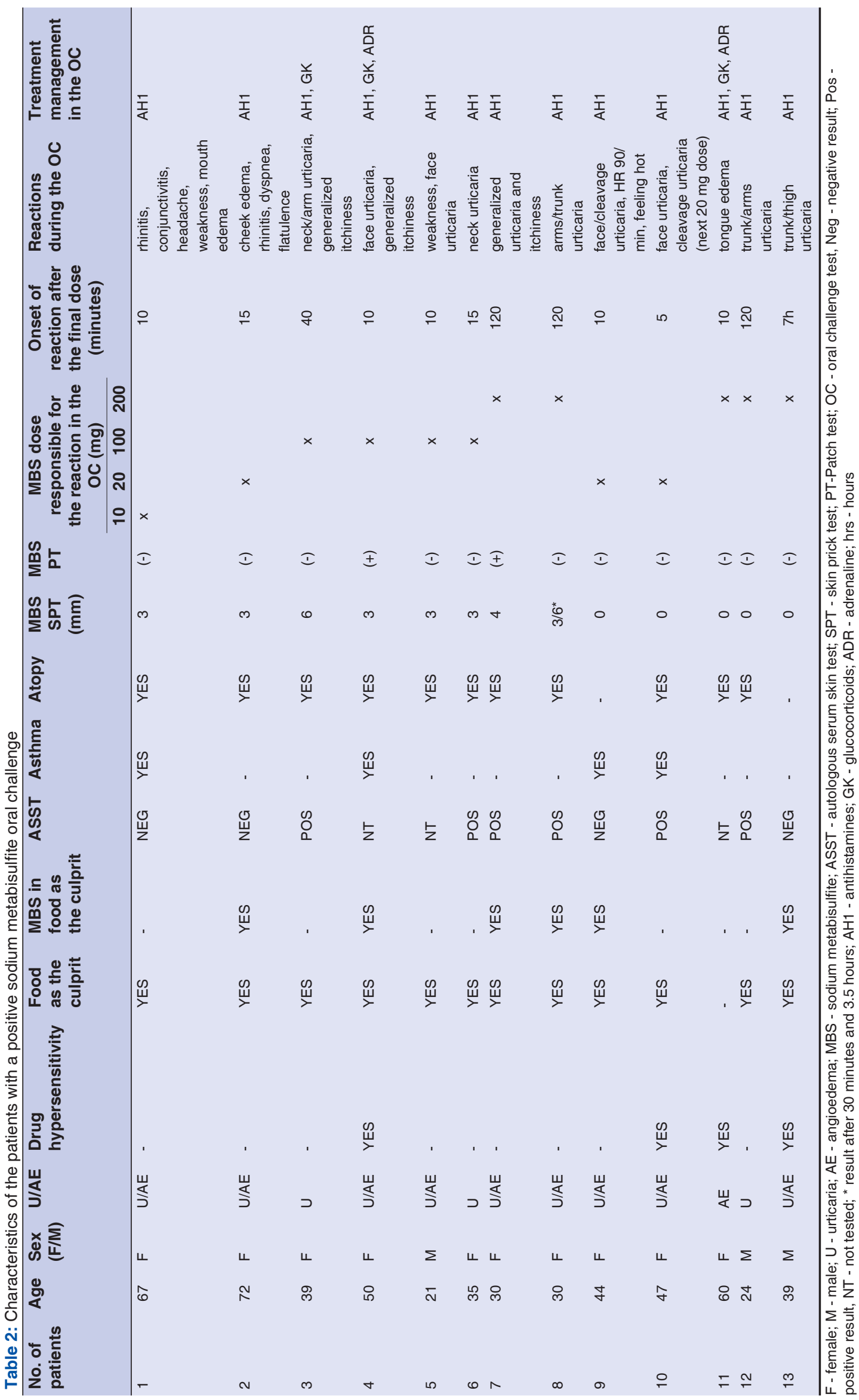


www.odermatol.com

Table 3: Skin prick test and patch test sensitivity and specificity in patients with sulfite hypersensitivity

\begin{tabular}{lcccccccc}
\hline & Sensitivity (\%) & Specificity (\%) & PPV (\%) & NPV (\%) & LR+ & LR- & OR & 95\% Cl \\
\hline SPT & 61.5 & 76.5 & 40.0 & 88.6 & 2.62 & 0.50 & 5.2 & $1.43-18.91$ \\
PT & 15.4 & 92.2 & 33.3 & 81.0 & 1.96 & 0.92 & 2.1 & $0.35-13.18$ \\
SPT or PT & 61.5 & 72.6 & 36.4 & 88.1 & 2.24 & 0.53 & 4.2 & $1.18-15.14$ \\
SPT and PT & 15.4 & 96.1 & 50.0 & 81.7 & 3.92 & 0.88 & 4.4 & $0.56-35.17$ \\
\hline
\end{tabular}

PPV - positive predictive value; NPV - negative predictive value; LR - likelihood ratio; OR - odds ratio; SPT - skin prick test; PT - patch test

Asero described a thirty-year-old female, in whom severe pruritus was observed after four hours of the administration of $10 \mathrm{mg}$ sodium metabisulfite [31]. In our study, paroxysmal pruritus associated with urticaria was also the main ailment in a thirty-year-old female patient (No. 7), who was refractory to treatment even with four antihistamines tablets daily. After stopping consuming muesli with dried fruits for breakfast, she gradually reduced the treatment to only one tablet per week. Our study showed that subsequent sulfite elimination correlated with the improvement of urticaria in five patients with a positive challenge.

According to the European Food Safety Authority (EFSA), the most sulfite-sensitive individuals may show reaction to ingested metabisulfite in quantities ranging from $20 \mathrm{mg}$ to $50 \mathrm{mg}$ [5]. Reports show that a single restaurant meal may contain 20-200 mg of sulfites [32], and their prohibited addition to products, such as lettuce or fruit salads, has been demonstrated [18,20]. The present study observed mouth edema with rhinitis, conjunctivitis, and headache in female patient No. 1 with asthma and a positive MBS SPT, occurring within 10 minutes of administering a $10 \mathrm{mg}$ dose of MBS. In addition, tearing, watery nasal discharge, and mild shortness of breath were seen up to 10 minutes after the application of a skin prick test, with the symptoms resolving spontaneously after 30 minutes; the patient had an associated airborne reaction after the exposure to sodium metabisulfite.

The results of the current study may be compared to those obtained by Ban et al. [9], who used doses ranging from $40 \mathrm{mg}$ to $200 \mathrm{mg}$ for the oral challenge. The authors showed that, in the group with urticaria and MBS hypersensitivity, the dose causing wheals during a challenge was, on average, $188 \mathrm{mg}$ after 100 minutes of the first dose. Our study determined that the average dose followed by symptoms was $113 \mathrm{mg}$, with the reaction time of 164 minutes of the first dose of MBS.

We ascertained a percentage of $5.5 \%(n=6)$ of positive sodium metabisulfite PTs, which was consistent with other studies $(2.2-7 \%)$ performed in the last ten years $[3,7,33-35]$. As the reaction was more prominent on D3 and D4 than on D2, we excluded irritancy. Similarly to some studies [20], we detected more positive MBS patch tests in females than males (5:1 ratio). However, we were not able to establish the primary source of sulfite sensitization.

We noticed that urticarial symptoms were relatively more severe in the MBS PT $(+)$ patients and lasted longer than in the rest. Similarly to other studies $[7,11]$, we determined that the patients with sulfite contact allergies may also have shown symptoms after their ingestion and that patch tests may be useful to diagnose immediate reactions to sulfite-containing foods [7,20]. Our study found two patients (No. 4 and 10) with a positive sodium metabisulfite SPT, PT, and challenge test. Table 3 shows the sensitivity and specificity of skin tests, wherein a positive oral challenge was regarded as the gold standard.

No group with a positive MBS test differed in terms of the coexistence of allergic rhinitis, dermatitis, asthma, or drug hypersensitivity. We found that sulfite hypersensitivity usually accompanies other allergic diseases and is rarely an isolated complaint.

Our study concluded that sodium metabisulfite hypersensitivity appears often enough to be screened in chronic urticaria and that more than half of the diagnosed urticarial cases were IgE-dependent reactions, sometimes coexisting with positive sodium metabisulfite patch tests.

\section{ACKNOWLEDGMENTS}

The authors would like to acknowledge Mikołaj Rybaczuk, M.A., for statistical analysis.

\section{Statement of Human and Animal Rights}

All the procedures followed were in accordance with the ethical standards of the responsible committee on human experimentation (institutional and national) and with the 2008 revision of the Declaration of Helsinki of 1975. 


\section{Statement of Informed Consent}

Informed consent for participation in this study was obtained from all patients.

\section{REFERENCES}

1. Kaaman AC, Boman A, Wrangsjö K, Matura M. Contact allergy to sodium metabisulfite: an occupational problem. Contact Dermatitis. 2010;63:110-2.

2. Madan V, Walker SL, Beck MH. Sodium metabisulfite allergy is common but is it relevant? Contact Dermatitis. 2007;57:173-6.

3. Oliphant T, Mitra A, Wilkinson M. Contact allergy to sodium sulfite and its relationship to sodium metabisulfite. Contact Dermatitis. 2012;66:128-30.

4. Steiner M, Scaife A, Semple S, Hulks G, Ayres JG. Sodium metabisulfite induced airways disease in the fishing and fishprocessing industry. Occup Med. 2008;58:545-50.

5. EFSA ANS Panel (EFSA Panel on Food Additives and Nutrient Sources Added to Food). Scientific opinion on the re-evaluation sulfur dioxide (E 220), sodium sulfite (E 221), sodium bisulfite (E 222), sodium metabisulfite (E 223), potassium metabisulfite (E 224), calcium sulfite (E 226), calcium bisulfite (E 227) and potassium bisulfite (E 228) as food additives. EFSA J. 2016;14:4438.

6. Uriarte SA, Fernández-Nieto M, Arochena L, Sastre J. Occupational asthma in seafood manufacturing and food allergy to seafood. J Investig Allergol Clin Immunol. 2015;25:59-60.

7. Garcia-Gavin J, Parente J, Goossens A. Allergic contact dermatitis caused by sodium metabisulfite: A challenging allergen: A case series and literature review. Contact Dermatitis. 2012;67:260-9.

8. Arai Y, Muto H, Sano Y, Ito K. Food and food additives hypersensitivity in adult asthmatics. III. Adverse reaction to sulfites in adult asthmatics. Arerugi. 1998;47:1163-7.

9. Ban GY, Kim MA, Yoo HS, Ye YM, Park HS. Letter to the editor. Two major phenotypes of sulfite hypersensitivity: Asthma and urticaria. Yonsei Med J. 2014;55:542-4.

10. Vally H, Thompson PJ. Role of sulfite additives in wine induced asthma: Single dose and cumulative dose studies. Thorax. 2001;56:763-9.

11. Garcia-Ortega P, Scorza E, Teniente A. Basophil activation test in the diagnosis of sulfite-induced immediate urticaria. Clin Exp Allergy. 2010;40:688.

12. Di Lorenzo G, Pacor ML, Mansueto P, Martinelli N, EspositoPellitteri M, Lo Bianco C, et al. Food-additive-induced urticaria: A survey of 838 patients with recurrent chronic idiopathic urticaria. Int Arch Allergy Immunol. 2005;138:235-42.

13. Belchi-Hernandez J, Florido-Lopez JF, Estrada-Rodriguez JL, Martinez-Alzamora F, Lopez-Serrano C, Ojeda-Casas JA. Sulfiteinduced urticaria. Ann Allergy. 1993;71:230-2.

14. Boyd AH, Warshaw EM. Sulfites: No Longer a Zebra? Dermatitis. 2017;28:364-6.

15. Cussans A, McFadden J, Ostlere L. Systemic sodium metabisulfite allergy. Contact Dermatitis. 2015;73:316-7.

16. Guha-Niyogi B, Sabroe R, Holden C. An unusual case of a systemic delayed hypersensitivity reaction to sodium metabisulfite. Contact Dermatitis. 2018;79:246-7.

17. Silva M, Gama J, Pinto N, Pivi G, Brancal H, Carvalho L, et al.
Sulfite concentration and the occurrence of headache in young adults: A prospective study. Eur J Clin Nutr. 2019;73:1316-22.

18. Yang WH, Purchase EC, Rivington RN. Positive skin tests and Prausnitz-Kustner reactions in metabisulfite-sensitive subjects. J Allergy Clin Immunol. 1986;78:443-9.

19. Vitaliti G, Guglielmo F, Giunta L, Pavone P, Falsaperla R. Sodium metabisulfite allergy with multiple food and drug hypersensitivities in a five-year-old child: A case report and literature review. Allergol Immunopathol. 2015;43:106-8.

20. Vally H, Misso NL, Madan V. Clinical effects of sulfite additives. Clin Exp Allergy. 2009;39:1643-51.

21. García Ortiz JC, Vega Gutiérrez JM, Pérez Velesar MJ, Medina AA. Occupational allergic contact dermatitis from potassium metabisulfite. Dermatitis. 2014;25:150-1.

22. Häberle M, Geier J, Mahler V. Contact allergy and intolerance to sulfite compounds: clinical and occupational relevance. Allergo J Int. 2017;26:53-66.

23. Seitz CS, Bröcker EB, Trautmann A. Eyelid dermatitis due to sodium metabisulfite. Contact Dermatitis. 2006;55:249-50.

24. Vally H, Misso NL. Adverse reactions to the sulfite additives. Gastroenterol Hepatol Bed Bench. 2012;5:16-23.

25. Schroecksnadel S, Jenny M, Fuchs D. Sensitivity to sulfite additives. Clin Exp Allergy. 2010;40:688-9.

26. Stingeni L, Bianchi L, Lisi P. Occupational airborne allergic contact dermatitis from potassium metabisulfite. Contact Dermatitis. 2009;60:52-3.

27. Habenicht HA, Preuss L, Lovell RG. Sensitivity to ingested metabisulfites: Cause of bronchospasm and urticaria. Immunol Allergy Pract. 1983;5:243-5.

28. Jimenez-Aranda GS, Flores-Sandoval G, Gomez-Vera J, OreaSolano M. Prevalence of chronic urticaria following the ingestion of food additives in a third tier hospital. Rev Alerg Mex. 1996;43:152-6.

29. Montaño García ML, Orea M. Frequency of urticaria and angioedema induced by food additives. Rev Alerg Mex. 1989;36:15-8.

30. Rajan JP, Simon RA, Bosso JV. Prevalence of sensitivity to food and drug additives in patients with chronic idiopathic urticaria. J Allergy Clin Immunol Pract. 2014;2:168-71.

31. Asero R. Food additive-induced chronic pruritus: further evidence. Clin Exp Dermatol. 2005;30:719-20.

32. Settipane GA. Adverse reactions to sulfites in drugs and foods. J Am Acad Dermatol. 1984;10:1077-80.

33. Catli G, Bostanci I, Ozmen S, Dibek Misirlioglu E, Duman H, Ertan U. Is patch testing with food additives useful in children with atopic eczema? Pediatr Dermatol. 2015;32:684-9.

34. Kręcisz B, Chomiczewska-Skóra D, Kieć-Świerczyńska M. Preservatives as important etiologic factors of allergic contact dermatitis. Med Pr. 2015;66:327-32.

35. Ralph N, Verma S, Merry S, Lally A, Kirby B, Collins P. What is the relevance of contact allergy to sodium metabisulfite and which concentration of the allergen should we use? Dermatitis. 2015;26:162-5

Copyright by Beata Sadowska, et al. This is an open access article distributed under the terms of the Creative Commons Attribution License, which permits unrestricted use, distribution, and reproduction in any medium, provided the original author and source are credited.

Source of Support: Nil, Conflict of Interest: None declared. 